\title{
Rerare of Lista de anomalias congênitas prioritárias para vigilância no âmbito do Sistema de Informaç̧ões sobre Nascidos Vivos do Brasil
}

doi: 10.1590/S1679-49742021000100030

\author{
List of priority congenital anomalies for surveillance under the Brazilian Information System \\ on Live Births
}

\section{Lista de anomalías congénitas prioritarias para la vigilancia bajo el Sistema de Información sobre Nacidos Vivos en Brasil}

\author{
Augusto César Cardoso-dos-Santos ${ }^{1}$ - (D orcid.org/0000-0002-1499-9105 \\ Ana Cláudia Medeiros-de-Souza' - Đ orcid.org/0000-0001-9317-6424 \\ João Matheus Bremm ${ }^{1}$ - Đ orcid.org/0000-0002-2150-9426 \\ Ronaldo Fernandes Santos Alves ${ }^{1}$ - (1) orcid.org/0000-0002-8358-0519 \\ Valdelaine Etelvina Miranda de Araújo' ${ }^{1}$ - (D orcid.org/0000-0003-1263-1646 \\ Júlio Cesar Loguercio Leite ${ }^{2}$ - (๑ orcid.org/0000-0003-3858-8949 \\ Lavínia Schuler-Faccini ${ }^{2}$ - ๑ orcid.org/0000-0002-2428-0460 \\ Maria Teresa Vieira Sanseverino ${ }^{3}$ - (1) orcid.org/0000-0002-7404-2911 \\ Simone de Menezes Karam ${ }^{4}$ - (1) orcid.org/0000-0003-4413-1494 \\ Têmis Maria Félix ${ }^{2}$ - Đ orcid.org/0000-0002-8401-6821 \\ Mariana Bertol Leal ${ }^{5}$ - (1) orcid.org/0000-0002-1453-6100 \\ Eduardo Marques Macário' - (1) orcid.org/0000-0002-6383-0365 \\ Arnaldo Correia de Medeiros ${ }^{1}$ - (1) orcid.org/0000-0001-7341-0017 \\ Giovanny Vinícius Araújo de França' ${ }^{1}$ - Đorcid.org/0000-0002-7530-2017 \\ ${ }^{1}$ Ministério da Saúde, Secretaria de Vigilância em Saúde, Brasília, DF, Brasil \\ ${ }^{2}$ Hospital de Clínicas de Porto Alegre, Serviço de Genética Médica, Porto Alegre, RS, Brasil \\ ${ }^{3}$ Pontifícia Universidade Católica do Rio Grande do Sul, Escola de Medicina, Porto Alegre, RS, Brasil \\ ${ }^{4}$ Universidade Federal do Rio Grande, Faculdade de Medicina, Rio Grande, RS, Brasil \\ ${ }^{5}$ Ministério da Saúde, Secretaria de Ciência, Tecnologia e Insumos Estratégicos, Brasília, DF, Brasil
}

\section{Resumo}

Objetivo: Definir a lista de anomalias congênitas prioritárias para o aprimoramento do registro no Sistema de Informações sobre Nascidos Vivos (Sinasc). Métodos: A partir da Décima Revisão da Classificação Estatística Internacional de Doenças e Problemas Relacionados à Saúde (CID-10), protocolos internacionais e reuniões com especialistas, a lista de anomalias prioritárias foi construída considerando-se dois critérios principais: ser diagnosticável ao nascimento; e possuir intervenção disponível em diferentes níveis. A lista foi submetida a apreciação da Sociedade Brasileira de Genética Médica e Genômica. Resultados: Compuseram a lista oito grupos de anomalias congênitas distribuídos de acordo com o tipo de anomalia relacionada, bem como a parte do corpo afetada e sua correspondência ao código do capítulo XVII da CID-10. Conclusão: A lista de anomalias congênitas prioritárias para notificação fornece subsídios para o aprimoramento do registro no Sinasc.

Palavras-chave: Anormalidades Congênitas; Serviços de Saúde; Sistemas de Informação; Monitoramento Epidemiológico; Diretório.

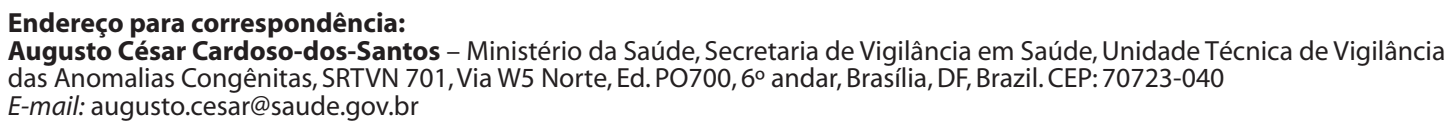




\section{Introdução}

Anomalias congênitas compreendem uma ampla gama de alterações estruturais ou funcionais, de origem pré-natal. ${ }^{1,2}$ No mundo, estima-se que aproximadamente 303 mil recém-nascidos morrem por ano nas primeiras quatro semanas de vida, como decorrência de anomalias congênitas, constituindo a principal causa de mortalidade infantil em países de renda alta. ${ }^{1}$ No Brasil, as anomalias congênitas representaram a segunda causa de morte em crianças com menos de 5 anos de idade no ano de 2017. ${ }^{3,4}$ O Sistema de Informações sobre Nascidos Vivos (Sinasc) é o sistema oficial para registro de anomalias congênitas estruturais visíveis no momento do parto, para todo recém-nascido em território nacional. ${ }^{5}$

\section{No Brasil, as anomalias congênitas representaram a segunda causa de morte em crianças com menos de 5 anos de idade no ano de 2017.}

A cada ano, cerca de 25 mil nascidos vivos são registrados no Sinasc com algum tipo de anomalia congênita. ${ }^{4}$ Todavia, existe uma notória heterogeneidade espaço-temporal na notificação das anomalias, nacionalmente. $\mathrm{Na}$ maioria dos casos, tal variabilidade é atribuída ao sub-registro ou registro errôneo de determinados tipos de anomalias, dificultando a realização de análises epidemiológicas fidedignas a partir dos dados do sistema supracitado ${ }^{4,-8}$ No intuito de aprimorar a qualidade dos registros de anomalias no Sinasc, em discussão com especialistas, identificou-se a necessidade de criar um rol de anomalias prioritárias para registro ao nascimento. Isto porque algumas anomalias não são visíveis na hora do parto e outras, embora diagnosticáveis ao nascimento, exigem instrumentos ou conhecimentos técnicos específicos que nem sempre se encontram disponíveis em todos os serviços públicos de saúde ou de assistência suplementar à saúde. ${ }^{6,9}$

Em se tratando da coleta de dados com propósitos de vigilância da Saúde Pública, a qualidade é tão importante como a quantidade. Inclusive, dados de alta qualidade sobre a frequência de algumas anomalias selecionadas podem ser mais úteis que dados de baixa qualidade sobre todas as anomalias. ${ }^{2}$ Destaforma, a lista prioritária deve ser composta por anomalias diagnosticáveis ao nascimento, de modo a produzir indicadores epidemiológicos mais fidedignos sobre a temática no país e, sob o ponto de vista de Saúde Pública, oferecer-se alguma intervenção possível.

0 presente trabalho teve por objetivo relatar o processo de construção da lista de anomalias congênitas prioritárias para vigilância no âmbito do Sinasc.

\section{Métodos}

Trata-se de pesquisa documental que partiu da Décima Revisão da Classificação Estatística Internacional de Doenças e Problemas Relacionados à Saúde (CID-10), protocolos internacionais ${ }^{9,10}$ e reuniões com especialistas, com vistas a estabelecer uma lista de anomalias congênitas prioritárias para notificação via Sinasc. Os principais protocolos utilizados foram o 'Manual para Gestores de Programa de Vigilância dos Defeitos Congênitos' e o 'Atlas de Anomalias Selecionadas', ambos da coleção 'Vigilância de Defeitos Congênitos' (ou Birth Defects Surveillance), construídos com a parceria da Organização Mundial da Saúde (OMS), Centro de Controle e Prevenção de Doenças dos Estados Unidos (CDC/USA) e International Clearinghouse for Birth Defects Surveillance and Research (ICBDSR) - uma rede internacional com o objetivo de promover vigilância e pesquisa de anomalias congênitas com base em diferentes registros ao redor do mundo. ${ }^{9,10}$

Desde 1990, o Sinasc coleta dados sobre os nascimentos em todo o território nacional, registrados na Declaração de Nascido Vivo (DNV); e desde 1999, também capta informações sobre anomalias congênitas estruturais. ${ }^{5}$ Além de informações sobre anomalias, a DNV apresenta dados antropométricos e sociodemográficos da mãe e da criança, dados gestacionais, data e local de ocorrência do parto, local de residência da mãe, entre outros.

A Lei $n^{0} 12.662$, de 5 de junho de 2012, transformou a DNV em documento de identidade provisório, assegurando sua validade nacional, enquanto a Lei $\mathrm{n}^{\circ}$ 13.685, de 25 de junho de 2018, estabeleceu que as informações sobre anomalias identificadas no momento do nascimento sejam registradas na DNV. Por conseguinte, a utilização de dados contidos na DNV, enquanto fonte primária de informação sobre anomalias congênitas, encontra respaldo legal e cobertura de praticamente toda a população de nascidos vivos no país. ${ }^{11}$ 
0 registro de anomalias no Sinasc pauta-se no preenchimento da DNV, tendo-se, como referência, os códigos presentes no capítulo XVII - Malformações congênitas, deformidades e anomalias cromossômicas - da CID-10.5 A seleção das anomalias a serem consideradas prioritárias para notificação via Sinasc foi realizada com base nesse capítulo, o qual dispõe 619 códigos associados a anomalias congênitas. ${ }^{12}$ As anomalias encontradas fora do capítulo XVII ou que não estivessem associadas a um código específico da CID-10 foram excluídas da seleção.

Foram realizadas três reuniões presenciais, entre técnicos do Ministério da Saúde e especialistas na temática, no período de 2019-2020. 0 grupo de especialistas foi formado por profissionais ligados ao Estudo Latino-Americano de Malformações Congênitas e a instituições de pesquisa e assistência à saúde do Rio Grande do Sul.

Nessas reuniões, à luz de protocolos internacionais para vigilância das anomalias congênitas, ${ }^{9,10}$ foi estabelecida uma lista preliminar de anomalias a serem consideradas prioritárias para notificação, considerando-se dois critérios principais para a seleção das anomalias congênitas: ser diagnosticável ao nascimento; e possuir intervenção preventiva em diferentes níveis. Ambos os critérios são detalhados a seguir.

a) Diagnosticável ao nascimento

As anomalias congênitas diagnosticáveis ao nascimento são, em grande parte, aquelas conhecidas como 'maiores' (ou maior), foco da maior parte dos programas de vigilância ao redor do mundo. Elas são definidas como alterações estruturais que implicam importantes consequências médicas, sociais ou cosméticas para os indivíduos afetados e, normalmente, requerem assistência médica. Não é raro uma anomalia maior vir acompanhada de anomalias menores, ou seja, outras alterações que não interferem significativamente na saúde dos indivíduos, cujas consequências sociais ou estéticas são limitadas., ${ }^{2,9}$

Além de serem potencialmente detectáveis ao nascimento, é importante que as anomalias sejam facilmente diagnosticáveis nesse momento, isto é, que de sua detecção possa se encarregar uma ampla gama de profissionais de saúde e não apenas especialistas em dismorfologia; e que não exija estruturas complexas para sua realização. A lista de anomalias prioritárias foi elaborada considerando-se essas limitações, haja vista as informações epidemiológicas resultantes da vigilância nacional poderem ser diretamente influenciadas por questões ligadas ao diagnóstico das alterações.
Tal proposição baseou-se na limitação existente na rede assistencial para o diagnóstico precoce de algumas anomalias, pois o registro no Sinasc ocorre somente a partir dos dados coletados ainda na sala de parto dos serviços de saúde. No entanto, reconheceu-se a possibilidade de agravos - quando identificados em diagnóstico por imagem ao longo da gestação - serem objeto de olhar mais atento da equipe médica logo ao nascimento, para melhor se diagnosticar e, então, gerar o registro na DNV.

b) Intervenção preventiva em diferentes níveis

Diferentes tipos de anomalias podem ser evitados com medidas simples, relativamente pouco onerosas, como a suplementação nutricional de ácido fólico e iodo durante a gestação, vacinação materna e controle de problemas de saúde materna, como o diabetes mellitus; ou adoção de hábitos saudáveis, como abstenção do consumo de álcool e fumo (prevenção primária)..$^{1,13}$

A prevenção secundária no Brasil é limitada por lei. 0 diagnóstico pré-natal de anomalias, seguido de interrupção de gravidez, não é permitido a não ser em casos de anencefalia. Mesmo que o impacto das medidas de prevenção secundária seja pequeno, o diagnóstico pré-natal continua a ser importante. Ele permite intervenções precoces que salvam vidas, como no caso de cardiopatias congênitas, além de abrir possibilidades para o encaminhamento da gestante a centros de referência. ${ }^{13}$

Finalmente, como exemplos de prevenção terciária, encontram-se as cirurgias pediátricas para correção de fissuras orofaciais e defeitos cardíacos. Realizadas na janela de tempo adequada, essas intervenções cirúrgicas podem reduzir drasticamente a morbidade ou mesmo a mortalidade relacionada. Em consonância, métodos de estimulação precoce e reabilitação também podem ser contemplados como exemplos de intervenção preventiva terciária, com o objetivo de melhorar a qualidade de vida dos indivíduos com anomalias congênitas.

\section{Revisão da lista}

A lista preliminar foi submetida a apreciação da Sociedade Brasileira de Genética Médica e Genômica (SBGM), cujas considerações foram analisadas e incorporadas à lista final. A Figura 1 apresenta um fluxograma do processo resumido, das principais etapas do trabalho até o resultado final. 


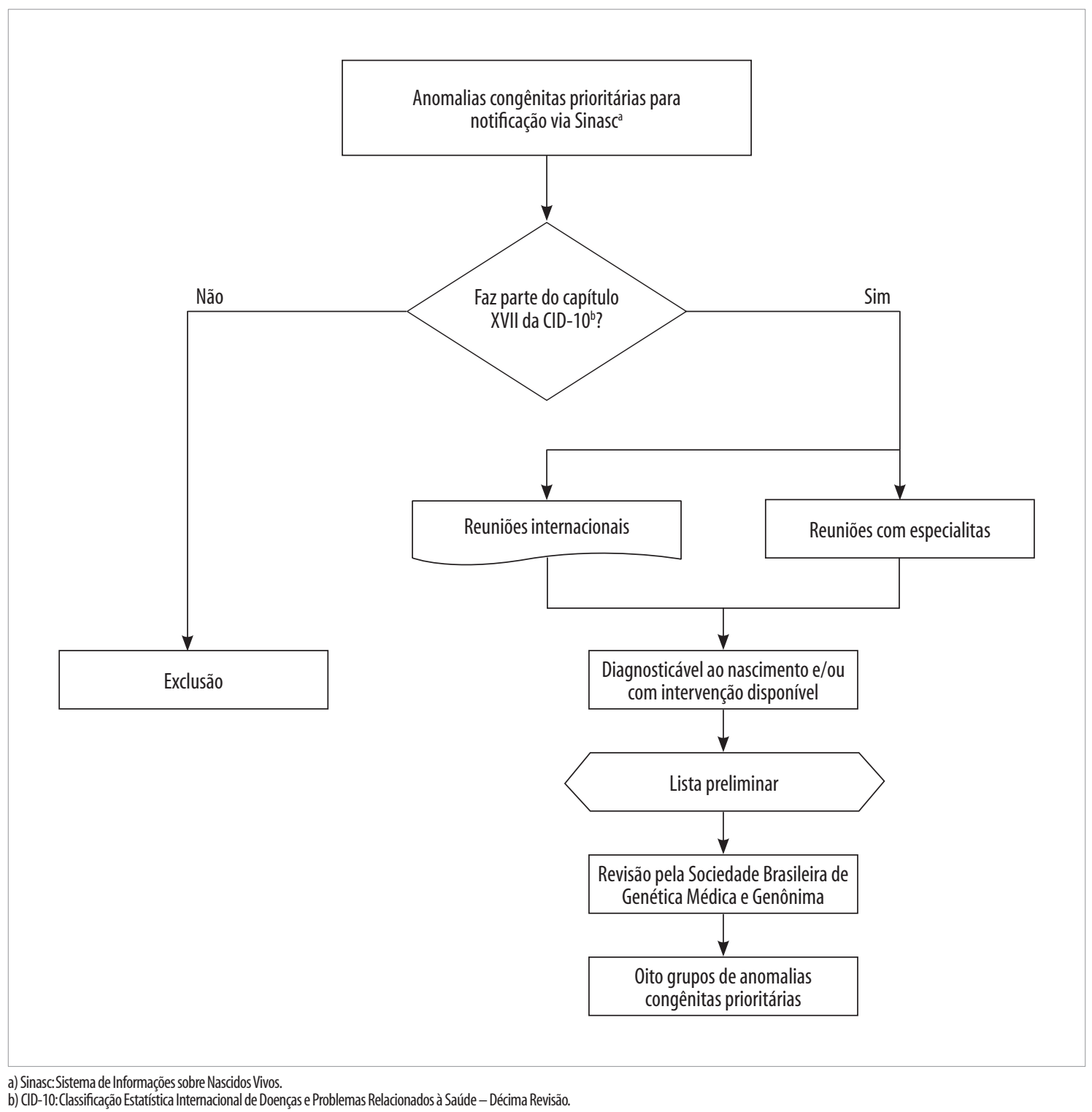

Figura 1 - Fluxograma de processo, destacando-se as principais etapas de construção da lista prioritária de anomalias congênitas para fortalecimento da notificação no Sinasc ${ }^{a}$

\section{Resultados}

Antes de mais nada, cumpre lembrar que a CID-10 reúne 898 entidades patológicas enquadráveis no conceito de anomalias congênitas, das quais 619 encontram-se listadas em seu capítulo XVII. ${ }^{12}$

Após aplicação dos critérios supracitados, a lista preliminar constituiu-se de sete grupos de anomalias congênitas, agrupadas conforme a distribuição hierárquica da CID-10. Inicialmente, considerou-se o tipo de anomalia relacionada, assim como a parte do corpo afetada, a saber: defeitos de tubo neural (Q00.0, Q00.1, Q00.2, Q01 e Q05); microcefalia (Q02); cardiopatias congênitas (Q20 a Q28); fendas orais (Q35 a Q37); anomalias de órgãos genitais (Q54 e Q56); defeitos de membros (Q71, Q72 e Q73); e defeitos de parede abdominal (Q79.2 e Q79.3). Logo, na etapa de revisão pela SBGM, sugeriu-se a inclusão 
de deformidades congênitas do pé (Q66), polidactilia (Q69) e artrogripose congênita múltipla (Q74.3) ao grupo 'defeitos de membros', além da criação de um novo grupo para síndrome de Down (Q90).

Todas as anomalias que compuseram a lista atenderam a ambos os critérios estabelecidos; à exceção das cardiopatias congênitas, cujo critério de ser facilmente diagnosticável ao nascimento nem sempre se pode aplicar. A lista completa de anomalias prioritárias para notificação no Sinasc é apresentada na Figura 2.

\begin{tabular}{|c|c|c|}
\hline Grupo de anomalias & Código da CID-10a & Descrição \\
\hline \multirow[t]{5}{*}{ Defeitos do tubo neural } & Q00.0 & Anencefalia \\
\hline & Q00.1 & Craniorraquisquise \\
\hline & 000.2 & Iniencefalia \\
\hline & Q01 & Encefalocele \\
\hline & Q05 & Espinha bífida \\
\hline Microcefalia & Q02 & Microcefalia \\
\hline \multirow[t]{9}{*}{ Cardiopatias congênitas } & Q20 & Malformações congênitas das câmaras e das comunicações cardíacas \\
\hline & Q21 & Malformações congênitas dos septos cardíacos \\
\hline & Q22 & Malformações congênitas das valvas pulmonar e tricúspide \\
\hline & Q23 & Malformações congênitas das valvas aórtica e mitral \\
\hline & Q24 & Outras malformações congênitas do coração \\
\hline & Q25 & Malformações congênitas das grandes artérias \\
\hline & Q26 & Malformaç̧ões congênitas das grandes veias \\
\hline & Q27 & Outras malformações congênitas do sistema vascular periférico \\
\hline & Q28 & Outras malformações congênitas do aparelho circulatório \\
\hline \multirow[t]{3}{*}{ Fendas orais } & Q35 & Fenda palatina \\
\hline & Q36 & Fenda labial \\
\hline & Q37 & Fenda labial com fenda palatina \\
\hline \multirow{2}{*}{ Anomalias de órgãos genitais } & Q54 & Hipospádia \\
\hline & Q56 & Sexo indeterminado e pseudo-hermafroditismo \\
\hline \multirow[t]{6}{*}{ Defeitos de membros } & Q66 & Deformidades congênitas do pé \\
\hline & Q69 & Polidactilia \\
\hline & Q71 & Defeitos, por redução, do membro superior \\
\hline & Q72 & Defeitos, por redução, do membro inferior \\
\hline & Q73 & Defeitos por redução de membro não especificado \\
\hline & Q74.3 & Artrogripose congênita múltipla \\
\hline \multirow{2}{*}{ Defeitos de parede abdominal } & Q79.2 & Exonfalia \\
\hline & 079.3 & Gastrosquise \\
\hline Síndrome de Down & Q90 & Síndrome de Down \\
\hline
\end{tabular}

a) CID-10:Classificação Estatística Internacional de Doenças e Problemas Relacionados à Saúde - Décima Revisão.

Figura 2 - Lista de anomalias congênitas prioritárias para propósitos de vigilância nacional, classificadas de acordo com a CID-10a, Brasil, 2020 


\section{Discussão}

A lista de oito grupos de anomalias congênitas prioritários, conforme definição deste trabalho, fornece subsídios para o aprimoramento do registro no Sinasc. Dadas as complexidades envolvidas no diagnóstico e notificação das anomalias com base no Sinasc, no contexto de um país social e demograficamente diverso, populoso e territorialmente vasto como o Brasil, poder dispor de uma lista de anomalias prioritárias, selecionadas com base em aspectos relevantes para seu reconhecimento e manejo, revelou-se uma aquisição de importância fundamental para o sistema.

Algumas limitações foram encontradas durante 0 processo de construção da lista prioritária, a exemplo do uso da CID-10 ao invés da nova versão da classificação internacional, a CID-11, que acrescentou um novo capítulo dedicado às anomalias congênitas: 0 capítulo XX, Anomalias do desenvolvimento, para inclusão de novos códigos. ${ }^{12}$ Todavia, optou-se por utilizar a CID-10 tendo em vista ser esta a versão em uso nos sistemas oficiais de informações em saúde do Brasil (especialmente no Sinasc), para registro das doenças e problemas de saúde. ${ }^{14,15}$ Outra possível limitação do trabalho seria a ausência de documentação do processo de votação para consenso, que permitisse catalogação e cálculos, e assim, minimização de subjetividades. Porém, as decisões tomadas aconteceram em reuniões presenciais com especialistas, após deliberações consensuais e aprovação final por todos os envolvidos. Ademais, a lista pode ser revista e atualizada: novos métodos de diagnóstico ou intervenção preventiva podem tornar elegíveis anomalias não incluídas, por não atenderem aos critérios estabelecidos nesta publicação.

As anomalias selecionadas estão de acordo com os principais modelos de vigilância desses agravos em todo 0 mundo. ${ }^{16} \mathrm{O}$ 'Manual para Gestores de Programa de Vigilância dos Defeitos Congênitos', elaborado pelo ICBDSR em parceria com a OMS e o $\mathrm{CDC}$, recomenda que as anomalias a serem incluídas em um novo programa de vigilância sejam inicialmente limitadas às estruturais, prontamente identificáveis e facilmente reconhecidas no exame físico ao nascimento. ${ }^{9,10} \mathrm{~A}$ lista aqui apresentada inclui todas as anomalias sugeridas (defeitos de tubo neural; fendas lábio-palatinas; anomalias dos órgãos genitais; defeitos de redução de membros e pé torto; onfalocele e gastrosquise), tendo-se adicionado mais três categorias consideradas relevantes para nosso sistema de saúde: microcefalia, cardiopatias congênitas e síndrome de Down.

A microcefalia (Q02), isto é, redução do perímetro cefálico inferior à média da população com a mesma idade gestacional e peso, é uma anomalia sentinela para infecções congênitas, especialmente infecção pelos vírus Zika e citomegalovírus. A forma grave da microcefalia compreende uma medida de perímetro cefálico três desvios-padrão abaixo da média para idade e sexo. A prevalência de microcefalia ao nascimento no Brasil, captada pelo Sinasc, variou de $0,53 / 10$ mil a $0,63 / 10$ mil nascidos vivos (NV) entre 2010 e 2014, passou a 5,83/10 mil NV em 2015 e alcançou um pico de 7,96/10 mil NV em 2016. Nesse mesmo ano, considerando-se apenas a região Nordeste, a prevalência de microcefalia ao nascer atingiu 13,42/10 mil NV. ${ }^{4}$ Tal variação pode ser explicada, em grande parte, pela epidemia de síndrome congênita associada à infecção pelo vírus Zika, sobretudo entre 2015 e 2016, com epicentro no Nordeste brasileiro. ${ }^{17}$ A prevenção desse agravo pode ocorrer tanto em nível primário, evitando-se o contato com o mosquito transmissor do agente viral, como em nível terciário, por meio de estimulação precoce, reabilitação, entre outras medidas.

As cardiopatias congênitas constituem uma exceção parcial ao critério de facilidade de diagnóstico ao nascimento. Entretanto, elas são causa importante de mortalidade pós-natal, fazem parte do Plano Nacional de Assistência à Criança com Cardiopatia Congênita (que recomendou às maternidades realizarem o teste do coraçãozinho) e, muitas vezes, podem ser detectadas antes do nascimento, no exame de ultrassonografia morfológica e pela ecocardiografia fetal; ${ }^{18}$ sendo assim, foram consideradas um indicador importante para vigilância, ainda que seja esperado sub-registro.

A síndrome de Down (Q90) foi incluída na lista porque, embora o diagnóstico na sala de parto nem sempre seja possível, trata-se da síndrome genética mais comum, com incidência de aproximadamente 1/600 nascidos vivos. ${ }^{19}$ Logo, a epidemiologia da síndrome de Down pode ser considerada um bom indicador de qualidade de registros ao nascimento; outrossim, seu diagnóstico precoce possibilita o encaminhamento para terapias de apoio e aconselhamento genético da família.

A lista aqui apresentada possui um foco bastante específico na vigilância ao nascimento, para registro 
no Sinasc, e foi construída com objetivo de ampliar o diagnóstico e a oferta de cuidados para prevenção, e procedimentos de reabilitação das crianças. ${ }^{913}$ Todavia, é importante pontuar que os profissionais envolvidos no reconhecimento e codificação de anomalias devem ser encorajados a continuar diagnosticando e registrando todas as anomalias presentes no capítulo XVII da CID-10, incluindo aquelas não listadas como prioritárias. Lembra-se, uma vez mais, que a lista pode ser revista, sempre que necessário e complementada com outros agravos, levando-se em consideração os critérios aqui expostos. A presente iniciativa deverá estimular os profissionais e os serviços de saúde para melhor identificar e oferecer os devidos cuidados aos recém-nascidos que apresentem alguma anomalia.

A ação articulada entre a vigilância e a atenção à saúde poderá contribuir para a elaboração de novas políticas públicas de saúde., ${ }^{4,20}$ Os serviços de saúde são unidades sentinelas da vigilância, e o trabalho da vigilância ajuda a compreender os problemas de saúde e qualificar as políticas e os serviços nelas previstos, visando fortalecer a rede assistencial e as diferentes estratégias de cuidado implementadas pelo Sistema

\section{Referências}

1. World Health Organization. Congenital anomalies [Internet]. [Geneva: WHO]; 2016 [acesso 28 jan. 2020]. Disponível em: https://www.who.int/newsroom/fact-sheets/detail/congenital-anomalies

2. Centers Disease Control and Prevention. External major congenital anomalies for monitoring [Page last reviewed: October 30, 2019]. In: Birth defects surveillance toolkit [Internet]. [Washington, DC]: CDC; 2019 [acesso 12 jul 2020]. Available from: https://www.cdc.gov/ncbddd/birthdefects/ surveillancemanual/facilitators-guide/module-4/ mod4-1.html

3. Instituto de Pesquisa Econômica Aplicada. ODS 3: Assegurar uma vida saudável e promover o bem-estar para todas e todos, em todas as idades. In: Objetivos de Desenvolvimento Sustentável [Internet]. Brasília, -DF; 2019 [acesso 10 jul. 2020]. Disponível em: http://www.ipea.gov.br/portal/publicacoes

4. Ministério da Saúde (BR), . Secretaria de Vigilância em Saúde. Anomalias congênitas no Brasil, 2010 a 2018: análise dos dados de sistemas de informação para o fortalecimento da vigilância e atenção em saúde. Brasília, DF: MS; 2020 [acesso 10 jul. 2020]. Boletim Epidemiológico. p. 19-36. Disponível em: https://www.saude.gov.br/images/pdf/2020/marco/27/ Boletim-epidemiologico-SVS-13.pdf
Único de Saúde. Finalmente, o foco de construção da presente lista, com a inclusão de um número específico de anomalias, também assume relevância estratégica, na medida em que deve subsidiar a publicação, pelo Ministério da Saúde, de normativas indispensáveis à estruturação de um projeto nacional de vigilância de anomalias congênitas, além de servir à elaboração de instruções claras e objetivas, dirigidas aos profissionais de saúde que realizam a notificação de anomalias congênitas no Sinasc.

\section{Contribuição dos autores}

Todos os autores contribuíram com a concepção, delineamento e revisão crítica relevante do conteúdo intelectual do manuscrito. Cardoso-dos-Santos AC, Medeiros de Souza AC, Bremm JM, Alves RFS, Araujo VEM, Leite JCL, Schuler-Faccini L, Sanseverino MTV, Karam SM, Félix TM, Leal MB, Macário EM, Medeiros AC e França GVA participaram da redação do trabalho. Todos os autores aprovaram a versão final e assumem responsabilidade por todos os aspectos do trabalho, incluindo a garantia de sua precisão e integridade.

5. Ministério da Saúde (BR), Secretaria de Vigilância em Saúde. Sistema de informações sobre nascidos vivos (SINASC) [Internet]. Brasília, DF: SVS; [data desconhecida, acesso 6 jan. 2021. Disponível em: http://svs.aids.gov.br/dantps/cgiae/sinasc/

6. Luquetti DV. Avaliação da qualidade das informações sobre anomalias congênitas do sistema de informações sobre nascidos vivos. Rio de Janeiro: Fundação Oswaldo Cruz; 2009.

7. Ministério da Saúde (BR). Como nascem os brasileiros: captação e prevalência das anomalias congênitas. In: Saúde Brasil 2018: uma análise da situação de saúde e das doenças e agravos crônicos: desafios e perspectivas. Brasília, DF: MS; 2019. p. 15-32.

8. Laurenti R, Siqueira AAF, Jorge MHPM, Gotlieb SLD, Pimentel EC. The importance of congenital malformations at birth. J Hum Growth Dev. 2014;24(3):328-38.

9. World Health Organization. Birth defects surveillance: a manual for programme managers. Geneva: WHO; 2014.

10. World Health Organization. Birth defects surveillance: atlas of selected congenital anomalies. Geneva: WHO; 2014. 
11. Pedraza DF. Qualidade do sistema de informações sobre nascidos vivos (Sinasc): análise crítica da literatura. Cienc Saude Colet. 2012;17(10): 2729-37. doi: https://doi.org/10.1590/\$141381232012001000021.

12. Bremm JM, Cardoso-dos-Santos AC, Magalhães VS, Medeiros-de-Souza AC, Alves RFS, Araujo VEM, et al. Anomalias congênitas na perspectiva da vigilância em saúde: compilação de uma lista com base na CID-10. Epidemiol Serv Saude. 2020;29(5):e2020164. Epub 4 dez. 2020. doi: https://doi.org/10.1590/s167949742020000500015 .

13. Christianson A, Howson CP, Modell B. March of Dimes: global report on birth defects. New York: MD; 2006.

14. Datasus (BR), Coordenação Geral de Informações e Análise Epidemiológica. Consolidação do Sistema de informações sobre nascidos vivos: 2011 [Internet]. Brasília, DF; 2011 [acesso 10 jul. 2020]. Disponível em: http://tabnet.datasus.gov.br/cgi/sinasc/Consolida_ Sinasc_2011.pdf

15. Coordenação-Geral de Informações e Análises Epidemiológicas (BR). Instruções para preenchimento da DN versão Jan 2011 [Internet]. [Brasília, DF: CGIAE; 2011 [acesso 19 feb. 2020]. Disponível em: http://svs.aids.gov.br/dantps/cgiae/ sinasc/documentacao/

16. Cardoso-dos-Santos AC, Magalhães VS, Medeirosde-Souza AC, Bremm JM, Alves RFS, Araujo VEM, et al. International collaboration networks for the surveillance of congenital anomalies: a narrative review. Epidemiol Serv Saude2020;29(4):e2020093. doi: https://doi.org/10.5123/s167949742020000400003 .

17. França GVA, Pedi VD, Garcia MHO, Carmo GMI, Leal MB, Garcia LP. Síndrome congênita associada à infecção pelo vírus Zika em nascidos vivos no Brasil: descrição da distribuição dos casos notificados e confirmados em 2015-2016. Epidemiol Serv Saude. 2018;27(2):e2017473. doi: https://doi.org/10.5123/ s1679-49742018000200014.

18. Ministério da Saúde(BR). Portaria n. 1.727, de 11 de julho de 2017. Aprova o plano nacional de assistência à criança com cardiopatia congênita. Brasília, DF: Diário Oficial da União 12 jul. 2017 [acesso 20 jul. 2020]. Disponível em: http://bvsms.saude.gov.br/bvs/ saudelegis/gm/2017/prt1727_12_07_2017.

19. Barros ALO, Barros AO, Barros GL M, Santos MTBR. Sobrecarga dos cuidadores de crianças e adolescentes com síndrome de down. Cienc Saude Colet. 2017];22(11):3625-34. doi: https://doi. org/10.1590/1413-812320172211.31102016.

20. Ministério da Saúde (BR), Secretaria de Vigilância em Saúde - Síndrome congênita associada à infecção pelo vírus Zika. Brasília, DF; 2019. 31 p. Boletim Epidemiológico. 


\section{Abstract}

Objective: To define the list of priority congenital anomalies for improving the registration in the Brazilian Live Birth Information System (Sinasc). Methods: Based on International Classification of Diseases, Tenth Revision (ICD-10), internation protocols and meetings with specialists, the list of priority anomalies was built considering two main criteria: being diagnosable at birth and having intervention available at different levels. The list was submitted for consideration by the Brazilian Society of Medical Genetics and Genomics. Results: The list comprised eight groups of congenital anomalies distributed according to the type of anomaly related, as well as the affect body part, all of which were related to some code of chapter XVII of ICD-10. Conclusion: The list of priority congenital anomalies for notification provides subsidies for improving registration at Sinasc.

Keywords: CongenitalAbnormalities; Health Services; Information Systems; Epidemiological Monitoring; Directory.

\section{Resumen}

Objetivo: Definir la lista de anomalias congénitas prioritarias para perfeccionar el registro en el Sistema de Información de Nacidos Vivos (Sinasc). Métodos: Con base en la Clasificación Internacional de Enfermedades, Décima Revisión (CIE-10), protocolos internacionales y reuniones con especialistas, la lista de anomalias prioritarias se construyó considerando dos criterios principales: ser diagnosticables al nacer y tener intervención disponible en diferentes niveles. La lista fue sometida a la consideración de la Sociedad Brasileña de Genética y Genómica Médica. Resultados: La lista comprendía ocho grupos de anomalías congénitas distribuidos según el tipo de anomalía relacionada, así como la parte del cuerpo afectada, todos ellos relacionados con algún código del capítulo XVII de la CIE-10. Conclusión: La lista de anomalías congénitas prioritarias para notificación proporciona subsidios para mejorar el registro en Sinasc.

Palabras clave: Anomalias Congénitas; Servicios de Salud; Sistemas de Información; Monitoreo Epidemiológico; Directorio.

Recebido em 05/10/2020

Aprovado em 11/02/2021 